\title{
Asociación de los índices neutrófilo/linfocito y linfocito/ proteína C reactiva con mortalidad por COVID-19
}

\author{
Alejandra Albarrán-Sánchez, ${ }^{1}$ Ricardo D. González-Ríos, ${ }^{1}$ Paolo Alberti-Minutti, ${ }^{1}$ Maura E. Noyola-García, ${ }^{1}$ \\ Carlos E. Contreras-García, ${ }^{1}$ Juan C. Anda-Garay, ${ }^{1}$ Luis E. Martínez-Ascencio, ${ }^{1}$ David J. Castillo-López, ${ }^{1}$ \\ Luis A. Reyes-Naranjo, ${ }^{1}$ Luis A. Guízar-García, ${ }^{1}$ Guillermo Flores-Padilla ${ }^{1}$ y Claudia Ramírez-Rentería ${ }^{2 *}$ \\ ${ }^{1}$ Servicio de Medicina Interna; ${ }^{2}$ Unidad de Investigación Médica de Enfermedades Endocrinas. Instituto Mexicano del Seguro Social, Centro Médico \\ Nacional Siglo XXI, Hospital de Especialidades, Ciudad de México, México
}

\section{Resumen}

Introducción: Los índices neutrófilo/linfocito (INL) y linfocito/proteína $C$ reactiva (ILR) se usan para predecir severidad y mortalidad en diversas infecciones. Objetivo: Establecer en México el mejor punto de corte de INL e ILR para predecir la mortalidad en pacientes hospitalizados por COVID-19. Método: Estudio transversal analítico de pacientes hospitalizados por COVID-19 grave en un hospital de especialidades. Resultados: Falleció $34 \%$ de 242 pacientes analizados. Los sujetos fallecidos tenían mayor edad (62 versus 51 años, $p<0.001$ ), mayor prevalencia de hipertensión arterial sistémica $>10$ años (59.4 versus $45.1 \%, p=0.022$ ), así como INL más alto (17.66 versus $8.31, p<0.001)$ e ILR más bajo $(0.03$ versus 0.06 , $p<0.002)$ respecto a quienes sobrevivieron. Los puntos de corte para predecir mortalidad fueron INL $>12$ e ILR $<0.03$. La combinación de INL e ILR tuvo sensibilidad de $80 \%$, especificidad de $74 \%$, valor predictivo positivo de $46.15 \%$, valor predictivo negativo de $93.02 \%$ y razón de momios de 11.429 para predecir la mortalidad. Conclusión: INL $>12$ e ILR< 0.03 son biomarcadores útiles para evaluar el riesgo de mortalidad en pacientes mexicanos con COVID-19 grave.

PALABRAS CLAVE: COVID-19. Mortalidad hospitalaria. Índice neutrófilo/linfocito.

\section{Association of neutrophil-to-lymphocyte and lymphocyte-to-C-reactive protein ratios with COVID-19-related mortality}

\begin{abstract}
Introduction: Neutrophil-to-lymphocyte (NLR) and lymphocyte-to-C-reactive protein (LCR) ratios are used to predict severity and mortality in various infections. Objective: To establish the best NLR and LCR cutoff point to predict mortality in patients hospitalized for COVID-19 in Mexico. Method: Analytical cross-sectional study of patients hospitalized for severe COVID-19 in a specialty hospital. Results: Out of 242 analyzed patients, $34 \%$ died. The deceased subjects were older (62 vs. 51 years; $p<0.001$ ), had a higher prevalence of $>10$ years with systemic arterial hypertension (59.4 vs. $45.1 \%, p=0.022)$, as well as a higher NLR (17.66 vs. 8.31, $p<0.001)$ and lower LCR $(0.03$ vs. $0.06, p<0.002]$ with regard to those who survived. The cutoff points to predict mortality were NLR $>12$ and $L C R<0.03$. The combination of NLR/LCR had a sensitivity of $80 \%$, specificity of $74 \%$, positive predictive value of $46.15 \%$, negative predictive value of $93.02 \%$ and an odds ratio of 11.429 to predict mortality. Conclusion: NLR $>12$ and $L C R<0.03$ are useful biomarkers to evaluate the risk of mortality in Mexican patients with severe COVID- 19.
\end{abstract}

KEY WORDS: COVID-19. In-hospital mortality. Neutrophil-to-lymphocyte ratio.

Correspondencia:

*Claudia Ramírez-Rentería

E-mail: clau.r2000@gmail.com

0016-3813/@ 2020 Academia Nacional de Medicina de México, A.C. Publicado por Permanyer. Este es un artículo open access bajo la licencia
Fecha de recepción: 26-07-2020

Fecha de aceptación: 04-08-2020

DOI: $10.24875 / G M M .20000525$
Gac Med Mex. 2020;156:563-568

Disponible en PubMed

www.gacetamedicademexico.com CC BY-NC-ND (http://creativecommons.org/licenses/by-nc-nd/4.0/). 


\section{Introducción}

El espectro clínico de la infección por SARS-CoV-2 varía desde infección asintomática, enfermedad leve del tracto respiratorio superior hasta neumonía viral grave con síndrome de insuficiencia respiratoria aguda (SIRA). ${ }^{1,2}$ Los factores que aumentan el riesgo de hospitalización por COVID-19 son edad mayor de 65 años (RM $=6.06$, IC $95 \%=3.98-9.22)$, sexo masculino (RM = 1.76, IC $95 \%=1.41-2.18, p<0.00001),{ }^{3-8}$ tener hipertensión arterial sistémica $(\mathrm{RM}=2.72, \mathrm{IC}$ $95 \%=1.60-4.64, p=0.0002)$, diabetes mellitus $(\mathrm{RM}=3.68, \mathrm{IC} 95 \%=2.68-5.03, \mathrm{p}<0.00001)$, enfermedades cardiovasculares (RM $=5.19$, IC $95 \%=3.25$ $8.29, \mathrm{p}<0.00001)$ y enfermedades respiratorias $(\mathrm{RM}=5.15, \mathrm{IC} 95 \%=2.51-10.57, \mathrm{p}<0.00001) ;{ }^{;, 10}$ la obesidad incrementa la probabilidad de requerir atención en una unidad de cuidados intensivos ( $R M=3.6$, IC $95 \%=2.5-5.3, p<0.0001)$ y de muerte $(\mathrm{RM}=5.1$, IC $95 \%=2.3-11.1, p<0.001) .^{11,12}$

Todos estos factores, frecuentes en México, ${ }^{13}$ han incrementado la demanda de atención hospitalaria, por lo que se requieren estrategias rápidas y económicas para clasificar tempranamente a los pacientes con mayor riesgo de muerte. Existen biomarcadores para predecir la gravedad de COVID-19.14-18 En COVID-19 se han usado los índices neutrófilo/linfocito $(\mathrm{INL})^{19-21}$ y linfocito/proteína C reactiva (ILR); ${ }^{22,23} \sin$ embargo, los puntos de corte son variables y no se han establecido para mortalidad en la población mexicana. Debido a su bajo costo y disponibilidad, estos marcadores podrían ser de utilidad en la clasificación de pacientes con enfermedad grave. Aun no está clara la utilidad de estos índices en pacientes con alto riesgo de fallecer por COVID-19 hospitalizados en México. Conocer esta información favorecerá una estrategia de clasificación de pacientes más eficiente y un mejor uso de los recursos.

El objetivo de la investigación que se presentan fue determinar las diferencias en los índices INL y ILR en pacientes hospitalizados por COVID-19 grave y establecer el mejor punto de corte para predecir mortalidad en México.

\section{Método}

Estudio transversal analítico de adultos hospitalizados por COVID-19 grave, cuyo diagnóstico fue confirmado por reacción en cadena de la polimerasa con transcriptasa reversa (RT-PCR) y que fueron hospitalizados entre marzo y junio de 2020 en el Hospital de Especialidades, Centro Médico Nacional Siglo XXI. Se definió como caso grave el paciente con fiebre, frecuencia respiratoria $>30$ /minuto, insuficiencia respiratoria y saturación de oxigeno $<93 \%$ a medio ambiente o que reuniera los criterios de SIRA según la clasificación de la Organización Mundial de la Salud. ${ }^{2}$ Previa firma de consentimiento informado por el paciente o familiar legalmente responsable, se obtuvieron datos del expediente clínico, respetando las recomendaciones de la Declaración de Helsinki para experimentación en humanos. Con la biometría hemática se calculó INL (relación de las células por microlitro) e ILR (relación de linfocitos por microlitro entre valor de PCR en $\mathrm{mg} / \mathrm{L}$ ), ambos en números absolutos. La información se obtuvo durante las primeras 24 horas del ingreso y fue registrada en una base de datos que fue analizada con el programa SPSS versión 23.

Se realizó prueba de normalidad de KolmogorovSmirnov. Las variables cualitativas se registraron con frecuencias y porcentajes y las cuantitativas, con medidas de tendencia central y dispersión conforme su distribución. El análisis bivariado de las variables cualitativas se realizó con chi cuadrada y prueba de Fisher y el de las variables cuantitativas, con t de Student y prueba de Wilcoxon. Se efectuó cálculo de razón de momios $(\mathrm{RM})$ de cada variable que presentó diferencias en el análisis bivariado y curva de ROC para delimitar el mejor punto de corte de los índices en estudio.

\section{Resultados}

Se analizaron los datos de 242 pacientes hospitalizados con COVID-19 grave, de los cuales $68.6 \%$ fue del sexo masculino. El promedio de edad fue de 55 años (rango intercuartílico de 43 a 65.5); 49 pacientes continuaban hospitalizados al momento de este análisis, 45.8 \% (111/242) egresó del hospital por mejoría y $34 \%$ (82/242) falleció. Las características de los dos últimos grupos se describen en la Tabla 1; los pacientes que fallecieron tuvieron mayor edad. Las principales comorbilidades en ambos grupos fueron diabetes (36.5\%), hipertensión (48.4\%) y cardiopatía (20.6\%). Los pacientes que fallecieron tenían más años de diagnóstico de hipertensión y presentaron diferencias significativas en la biometría hemática y reactantes de fase aguda, aumento del INL y descenso del ILR.

Los principales factores de riesgo en los pacientes que fallecieron en el análisis univariado fueron tener 
Tabla 1. Características clínicas y bioquímicas de pacientes hospitalizados por COVID-19 grave

\begin{tabular}{|c|c|c|c|c|c|}
\hline Característica & \multicolumn{2}{|c|}{ Alta por mejoría $(n=111)$} & \multicolumn{2}{|c|}{ Defunción ( $n=83$ ) } & $p$ \\
\hline \multirow[t]{2}{*}{ Edad en años (media y RIC) } & \multicolumn{2}{|c|}{$51(40-59)$} & \multicolumn{2}{|c|}{$62(50-71)$} & $<0.001$ \\
\hline & $n$ & $\%$ & $\mathrm{n}$ & $\%$ & \\
\hline Edad $>65$ años & 19 & 17.3 & 33 & 40 & 0.019 \\
\hline Sexo masculino & 74 & 67 & 63 & 76 & 0.162 \\
\hline Pacientes con diabetes & 37 & 33.3 & 37 & 44.6 & 0.111 \\
\hline Hipertensión arterial sistémica & 45 & 45.1 & 49 & 59.4 & 0.072 \\
\hline Uso de IECA & 21 & 19.1 & 17 & 20.8 & 0.662 \\
\hline Uso de ARA & 64 & 57.7 & 43 & 52.2 & 0.698 \\
\hline \multirow[t]{2}{*}{ Cardiopatía conocida } & 24 & 22.1 & 16 & 19.1 & 0.706 \\
\hline & \multicolumn{2}{|c|}{ Media (RIC) } & \multicolumn{2}{|c|}{ Media (RIC) } & \\
\hline Hemoglobina glucosilada (\%) & \multicolumn{2}{|c|}{$9.0(6.8-11.3)$} & \multicolumn{2}{|c|}{$7.5(6.2-9.6)$} & 0.189 \\
\hline Años con hipertensión arterial & \multicolumn{2}{|c|}{$3(0.6-10.5)$} & \multicolumn{2}{|c|}{$10(5-17)$} & 0.022 \\
\hline Índice tabáquico (paquetes/año) & \multicolumn{2}{|c|}{$3.6(0.2-18.8)$} & \multicolumn{2}{|c|}{$3(0.2-5)$} & 0.302 \\
\hline $\mathrm{IMC}\left(\mathrm{kg} / \mathrm{m}^{2}\right)$ & \multicolumn{2}{|c|}{$29.7(25.4-33.3)$} & \multicolumn{2}{|c|}{$28.7(24.1-35.1)$} & 1.000 \\
\hline 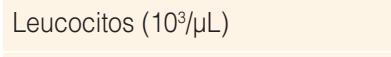 & \multicolumn{2}{|c|}{$7.51(5.70-11.50)$} & \multicolumn{2}{|c|}{$12.13(8.80-17.18)$} & $<0.001$ \\
\hline Neutrófilos $\left(10^{3} / \mu \mathrm{L}\right)$ & \multicolumn{2}{|c|}{$7.45(4.53-10.93)$} & \multicolumn{2}{|c|}{$8.98(6.80-14.71)$} & 0.014 \\
\hline Linfocitos $\left(10^{3} / \mu \mathrm{L}\right)$ & \multicolumn{2}{|c|}{$0.80(0.63-1.08)$} & \multicolumn{2}{|c|}{$0.60(0.39-0.90)$} & 0.002 \\
\hline Monocitos $\left(10^{3} / \mu \mathrm{L}\right)$ & \multicolumn{2}{|c|}{$0.30(0.21-0.44)$} & \multicolumn{2}{|c|}{$0.40(0.25-0.68)$} & 0.045 \\
\hline Plaquetas $\left(10^{3} / \mu \mathrm{L}\right)$ & \multicolumn{2}{|c|}{$227(165-326)$} & \multicolumn{2}{|c|}{$242(163-387)$} & 0.536 \\
\hline Hemoglobina (g/dL) & \multicolumn{2}{|c|}{$14.7(13.3-15.8)$} & \multicolumn{2}{|c|}{$14.3(11.8-15.8)$} & 0.559 \\
\hline Dímero D ( $\mu \mathrm{g} / \mathrm{mL})$ & \multicolumn{2}{|c|}{$0.91(0.64-1.55)$} & \multicolumn{2}{|c|}{$1.84(0.90-4.26)$} & 0.004 \\
\hline Fibrinógeno (mg/dL) & \multicolumn{2}{|c|}{$680(570-769)$} & 67 & & 0.924 \\
\hline Creatinina (mg/dL) & & & 1.0 & & $<0.001$ \\
\hline Deshidrogenasa láctica (U/L) & & & & & $<0.001$ \\
\hline Proteína $\mathrm{C}$ reactiva (mg/dL) & & & & & $<0.001$ \\
\hline Procalcitonina (ng/mL) & & & 1.7 & & $<0.001$ \\
\hline Ferritina (ng/mL) & & & 106 & & 0.357 \\
\hline Índice neutrófilo/linfocito & & & 17.66 & & $<0.001$ \\
\hline Índice linfocito/PCR & & & 0.03 & & 0.002 \\
\hline
\end{tabular}

hipertensión por más de 10 años, edad > 65 años, conteo leucocitario $\geq 12000$, dímero $\mathrm{D} \geq 1.0 \mu \mathrm{g} / \mathrm{mL}$, creatinina $\geq 1.2 \mathrm{mg} / \mathrm{dL}$, deshidrogenasa láctica $>300 \mathrm{U} / \mathrm{L}$, proteína $\mathrm{C}$ reactiva $\geq 21 \mathrm{mg} / \mathrm{L}$, procalcitonina $\geq 1.2 \mathrm{ng} / \mathrm{mL}$ y la combinación de INL elevado (> 12) e ILR bajo (<0.03), como se observa en la Tabla 2.
Se estableció un valor > 12 como punto de corte del INL para predecir mortalidad, con sensibilidad de $70.27 \%$, especificidad de $69.39 \%$, valor predictivo positivo de $63.41 \%$, valor predictivo negativo de $75.56 \%$ y precisión de $69.77 \%$, con un área bajo la curva de 0.728 . Los valores $<0.03$ del ILR se asociaron a mortalidad, con sensibilidad baja (51.7\%), pero 
Tabla 2. Análisis univariado de factores de riesgo para mortalidad por COVID-19 grave

\begin{tabular}{|c|c|c|c|}
\hline & Razón de momios (cruda) & IC $95 \%$ & p \\
\hline Sexo masculino & 0.610 & $0.229-1.624$ & 0.320 \\
\hline Hipertensión arterial sistémica > 10 años & 2.963 & $1-8.776$ & 0.047 \\
\hline Edad $>65$ años & 3.800 & $1.904-7.583$ & $<0.001$ \\
\hline Leucocitos > 12000 células/mL & 3.755 & $1.622-8.692$ & 0.002 \\
\hline Dímero $\mathrm{D}>1 \mu \mathrm{g} / \mathrm{mL}$ & 2.852 & $1.148-7.088$ & 0.022 \\
\hline Creatinina $>1.2 \mathrm{mg} / \mathrm{dL}$ & 3.097 & $1.616-5.936$ & 0.001 \\
\hline Albúmina $<3.5 \mathrm{gr} / \mathrm{dL}$ & 1.981 & $1.055-3.719$ & 0.032 \\
\hline Deshidrogenasa láctica > $300 \mathrm{U} / \mathrm{L}$ & 3.742 & $1.520-9.213$ & 0.003 \\
\hline Proteína C reactiva > 21 & 3.843 & $1.847-7.998$ & $<0.001$ \\
\hline Procalcitonina $>1.2$ & 3.768 & $1.682-8.442$ & 0.001 \\
\hline $\mathrm{INL}>12$ & 5.625 & $1.349-23.449$ & 0.021 \\
\hline ILR $<0.03$ & 8.786 & $2.70-28.593$ & 0.045 \\
\hline INL $>12-I L R<0.03$ & 11.429 & $2.807-46.528$ & 0.012 \\
\hline
\end{tabular}

especificidad alta $(89.13 \%)$ y valores predictivos adecuados (positivo de $75 \%$ y negativo de $74.55 \%$ ). La combinación de estos dos índices (INL e ILR) tuvo sensibilidad de $80 \%$, especificidad de $74 \%$, valor predictivo positivo de $46.15 \%$ y valor predictivo negativo de $93.02 \%$ para mortalidad.

\section{Discusión}

La clasificación rápida y apropiada de los pacientes con COVID-19 depende de herramientas sencillas, accesibles y baratas que permitan tomar decisiones clínicas en poco tiempo. Los INL y ILR pueden ser parte de las herramientas empleadas por los médicos que atienden a estos pacientes.

En un metaanálisis que incluyó seis estudios de China en los cuales se utilizaron los índices INL y ILR en pacientes con COVID-19, se observó que el INL aumentó de forma significativa en la forma grave de COVID-19 (media de 2.4, IC $95 \%$ = 0.98-3.82), mientras que el ILR disminuyó (media de 0.912, IC $95 \%=-1.275-0.550)$. Si bien ambos reflejaron el estado de inflamación y mal pronóstico en los pacientes con COVID-19, no se buscó un punto de corte específico para mortalidad. ${ }^{22}$ En un estudio de 21 hospitales de la provincia de Sichuan se encontró el valor > 9.8 como punto de corte para asociar el INL con mayor incidencia de SIRA y requerimiento de asistencia mecánica ventilatoria y que un INL de 11 constituía el punto de corte para demostrar SIRA moderada a severa (definida por un índice de oxigenación menor a 150), con área bajo la curva de 0.749 (IC $95 \%=0.624-0.850) . .^{20}$ En China, en una cohorte de 245 pacientes con COVID-19 se encontró que el INL es un factor de riesgo independiente para mortalidad hospitalaria, pero no fue posible determinar un punto de corte. ${ }^{24}$ En otros estudios se definió que un INL $\geq 3.3$ se asoció a severidad de la enfermedad y su valor predictor aumentó cuando la edad fue mayor de 49.5 años, si bien no se asoció a cambios en la mortalidad. ${ }^{19}$

En nuestra población, el INL > 12 se asoció a mortalidad, a diferencia de otros autores que lo han utilizado como predictor de severidad, lo cual probablemente explique el punto de corte más alto que los reportados con anterioridad. Todos los pacientes analizados tenían una forma grave de la enfermedad, en la cual es de gran importancia determinar la probabilidad de desenlace fatal.

Las diferencias en los puntos de corte reportados pueden tener otras causas. Debe considerarse que estos índices no son específicos para COVID-19. EI INL se eleva en enfermedades infecciosas, malignas, síndrome coronario agudo, hemorragia cerebral y dermatomiositis, ${ }^{25.26-28}$ mientras que el ILR se ha encontrado bajo en pacientes con cáncer colorrectal. ${ }^{29}$ 
Por otra parte, la población mexicana presenta con gran frecuencia obesidad y comorbilidades metabólicas, no comunes en China ni Europa. ${ }^{9,19,24}$ En nuestra serie, la hipertensión por sí misma no fue el factor más importante asociado a mortalidad, sino el tiempo de diagnóstico: más de 10 años fue un factor de riesgo importante. Lo anterior resalta la necesidad del diagnóstico oportuno de las comorbilidades y del seguimiento a largo plazo, así como la necesidad de programas preventivos de salud y evaluaciones periódicas especializadas.

Llama la atención que en este grupo particular de pacientes, el índice de masa corporal no fue distinto entre quienes murieron y quienes sobrevivieron. Esto puede deberse a que $83 \%$ de los pacientes estaba fuera del peso normal y la frecuencia de obesidad se distribuyó en forma similar entre los grupos, lo cual explica la alta frecuencia de pacientes que requirieron hospitalización y terapia intensiva, al igual que en otros reportes, pero no necesariamente representa mayor mortalidad si los pacientes reciben atención integral y tratamiento tanto por COVID-19 como por sus comorbilidades. Se identificó desnutrición en $4.5 \%$ de pacientes, condición que también se asocia a mortalidad elevada. Al parecer, las proporciones de obesidad, peso normal y desnutrición reflejan los índices de masa corporal de toda la población.

La edad fue mayor en el grupo de pacientes fallecidos y similar a la de otros reportes..$^{3-5}$

Dos limitaciones de este estudio estriban en que los datos fueron obtenidos retrospectivamente de los registros médicos y que fue realizado en un solo centro que atiende a población con enfermedades complejas y con múltiples comorbilidades, por lo que los resultados obtenidos pudieran no reflejar la realidad de otros centros hospitalarios.

Nuestros hallazgos indican la utilidad de INL e ILR en la práctica clínica cotidiana de médicos que atienden pacientes con COVID-19. Estos índices son fáciles de calcular y, además, son accesibles ya que se obtienen de los estudios de rutina solicitados al ingreso de los pacientes en la mayoría de los centros, incluso en aquellos con limitaciones importantes de recursos.

\section{Conclusión}

El INL $>12$ y el ILR $<0.03$ son biomarcadores accesibles y útiles para evaluar el riesgo de mortalidad por COVID-19 grave en mexicanos.

\section{Conflicto de intereses}

Los autores declaran no tener conflicto de intereses alguno.

\section{Financiamiento}

Los autores no recibieron patrocinio para llevar a cabo este artículo.

\section{Responsabilidades éticas}

Protección de personas y animales. Los autores declaran que los procedimientos seguidos se apegaron a las normas éticas del comité de experimentación humana responsable y a los lineamientos de la Asociación Médica Mundial y la Declaración de Helsinki.

Confidencialidad de los datos. Los autores declaran que siguieron los protocolos de su centro de trabajo sobre la publicación de datos de pacientes.

Derecho a la privacidad y consentimiento informado. Los autores obtuvieron el consentimiento informado de los pacientes o sujetos referidos en el artículo. Este documento obra en poder del autor de correspondencia.

\section{Bibliografía}

1. Guan WJ, Ni ZY, Hu Y, Liang WH, Ou CQ, He JX, et al. Clinical characteristics of coronavirus disease 2019 in China. N Engl J Med. 2020;382:1708-1720.

2. World Health Organization. Clinical management of severe acute respiratory infection (SARI) when COVID-19 disease is suspected: interim guidance. Suiza: World Health Organization; 2020.

3. Richardson S, Hirsch JS, Narasimhan M, Crawford, JM, McGinn T, Davidson KW, et al. Presenting characteristics, comorbidities, and outcomes among 5700 patients hospitalized with COVID-19 in the New York City Area. JAMA. 2020;323:2052-2059.

4. Petrilli CM, Jones SA, Yang J, Rajagopalan H, O'Donnell L, Chernyak Y, et al. Factors associated with hospital admission and critical illness among 5279 people with coronavirus disease 2019 in New York City: prospective cohort study. BMJ. 2020;369:m1966.

5. Chen T, Wu D, Chen H, Yan W, Yang D, Chen G. Clinical characteristics of 113 deceased patients with coronavirus disease 2019: retrospective study. BMJ. 2020;368: m1091.

6. Wu Z, McGoogan JM. Characteristics of and important lessons from the coronavirus disease 2019 (COVID-19) outbreak in China: summary of a report of 72314 cases from the Chinese Center for Disease Control and Prevention. JAMA. 2020;323:1239-1242.

7. Onder G, Rezza G, Brusaferro S. Case-fatality rate and characteristics of patients dying in relation to COVID-19 in Italy. JAMA. 2020;323: 1775-1776.

8. Severe outcomes among patients with coronavirus disease 2019 (COVID-19) - United States, February 12-March 16, 2020. MMWR Morb Mortal Wkly Rep 2020;69:343-346. DOI: http://dx.doi.org/10.15585/ mmwr.mm6912e2external icon

9. Zheng Z, Peng F, Xu B, Zhao J, Liu H, Peng J, et al. Risk factors of critical \& mortal COVID-19 cases: a systematic literature review and meta-analysis. J Infect. 2020;81:e16-e25.

10. Stokes EK, Zambrano LD, Anderson KN, Marder EP, Raz KM, Felix SEB, et al. Coronavirus disease 2019 case surveillance - United States, January 22-May 30, 2020. MMWR Morb Mortal Wkly Rep. 2020;69:759-765.

11. Lighter J, Phillips M, Hochman S, Sterling S, Johnson D, Francois F, et al. Obesity in patients younger than 60 years is a risk factor for COVID-19 hospital admission. Clin Infect Dis. 2020;71:896-897. 
12. Klang E, Kassim G, Soffer S, Freeman R, Levin MA, Reich DL. severe obesity as an independent risk factor for COVID-19 mortality in hospitalized patients younger than 50. Obesity. 2020;28:1595-1599.

13. Romero-Martínez M, Shamah-Levy T, Vielma-Orozco E, Heredia-Hernández $\mathrm{O}$, Mojica-Cuevas J, Cuevas-Nasu L, et al. Encuesta Naciona de Salud y Nutrición (Ensanut 2018): metodología y perspectivas. Salud Publica Mex. 2019;61:917-923.

14. Zhou F, Yu T, Du R, Fan G, Liu Z, Guan L. Clinical course and risk factors for mortality of adult inpatients with COVID-19 in Wuhan, China: a retrospective cohort study. Lancet. 2020;395:1054-1062.

15. Kermali M, Khalsa RK, Pillai K, Ismail Z, Harky A. The role of biomarkers in diagnosis of COVID-19 - a systematic review. Life Sci. 2020:254:117788.

16. Ji D, Zhang D, Xu J, Chen Z, Yang T, Zhao P, et al. Prediction for progression risk in patients with COVID-19 pneumonia: The CALL Score. Clin Infect Dis. 2020;71:1393-1399.

17. Wang D, Hu B, Hu C, Zhu F, Liu X, Zhang J, et al. Clinical characteristics of 138 hospitalized patients with 2019 novel coronavirus-infected pneumonia in Wuhan, China. JAMA. 2020;323:1061-1069.

18. Rogić $\mathrm{D}$, Juroš GF, Petrik J, Vrančić AL. Advances and pitfalls in using laboratory biomarkers for the diagnosis and management of sepsis. EJIFCC. 2017;28:114-121

19. Yang AP, Liu JP, Tao WQ, Li HM. The diagnostic and predictive role of NLR, d-NLR and PLR in COVID-19 patients. Int Immunopharmacol. 2020;84:106504.

20. Ponti G, Maccaferri M, Ruini C, Tomasi A, Ozben T. Biomarkers associated with COVID-19 disease progression. Crit Rev Clin Lab Sci. 2020:1-11.

21. Ma A, Cheng J, Yang J, Dong M, Liao X, Kang Y. Neutrophil-to-lymphocyte ratio as a predictive biomarker for moderate-severe ARDS in severe COVID-19 patients. Crit Care. 2020;24:288.
22. Lagunas-Rangel FA. Neutrophil-to-lymphocyte ratio and lymphocyte-to-C-reactive protein ratio in patients with severe coronavirus disease 2019 (COVID-19): a meta-analysis. J Med Virol. 2020.

23. Dolin HH, Papadimos TJ, Stepkowski S, Chen X, Pan ZK. A novel combination of biomarkers to herald the onset of sepsis prior to the manifestation of symptoms. Shock. 2018;49:364-370.

24. Liu Y, Du X, Chen J, Jin Y, Peng L, Wang HH, et al. Neutrophil-to-lymphocyte ratio as an independent risk factor for mortality in hospitalized patients with COVID-19. J Infec. 2020;81:e6-e12.

25. Azab B, Zaher M, Weiserbs KF, Torbey E, Lacossiere K, Gaddam S, et al. Usefulness of neutrophil to lymphocyte ratio in predicting short- and long-term mortality after non-ST-elevation myocardial infarction. Am J Cardiol. 2010;106:470-476.

26. Giede-Jeppe A, Bobinger T, Gerner ST, Sembill JA, Sprügel MI, Beuscher VD, et al. Neutrophil-to-Lymphocyte ratio is an independent predictor for in-hospital mortality in spontaneous intracerebral hemorrhage. Cerebrovascular Diseases 2017;44(1-2): 26-34. DOI: 10.1159/000468996

27. Ha YJ, Hur J, Go DJ, Kang EH, Park JK, Lee EY, et al. Baseline peripheral blood neutrophil-to-lymphocyte ratio could predict survival in patients with adult polymyositis and dermatomyositis: a retrospective observational study. PLoS One. 2018;13:e0190411.

28. Guthrie GJ, Charles KA Roxburgh CS, Horgan PG, McMillan DC Clarke SJ. The systemic inflammation-based neutrophil-lymphocyte ratio: experience in patients with cancer. Crit Rev Oncol Hematol. 2013;88: 218-230.

29. Okugawa Y, Toiyama Y, Yamamoto A, Shigemori T, Ide S, Kitajima T, et al. Lymphocyte-C-reactive protein ratio as promising new marker for predicting surgical and oncological outcomes in colorectal cancer. Ann Surg. 2020;272(2):342-351. DOI: 10.1097/SLA.0000000000003239 